\title{
Phase slips, dislocations, half-integer vortices, two-fluid hydrodynamics and the chiral anomaly in charge and spin density waves.
}

\author{
S. Brazovskii ${ }^{a, *}$, and N. Kirova ${ }^{b}$ \\ ${ }^{1}$ Université Paris-Saclay, CNRS, LPTMS, 91405, Orsay, France \\ ${ }^{2}$ Université Paris-Saclay, CNRS, LPS, 91405, Orsay, France \\ *e-mail: serguei.brazovski@universite-paris-saclay.fr \\ Contribution for the JETP special issue in honor of \\ I.E.Dzyaloshinskii's 90th birthday
}

\begin{abstract}
This brief review recalls some chapters in theory of sliding incommensurate density waves which may have appeared after inspirations from studies of I.E Dzyaloshinskii and collaborations with him. First we address the spin density waves which rich order parameter allows for an unusual object of a complex topological nature: a half-integer dislocation combined with a semi-vortex of the staggered magnetization. It becomes energetically preferable with respect to an ordinary dislocation due to the high Coulomb energy at low concentration of carriers. Generation of these objects should form a sequence of $\pi$ - phase slips in accordance with experimental doubling of phase-slips rate.

Next, we revise the commonly employed TDGL approach which is shown to suffer from a violation of the charge conservation law resulting in nonphysical generation of particles which is particularly pronounced for electronic vortices in the course of their nucleation or motion. The suggested consistent theory exploits the chiral transformations taking into account the principle contribution of the fermionic chiral anomaly to the effective action. The derived equations clarify partitions of charges, currents and rigidity among subsystems of the condensate and normal carriers and the gluing electric field. Being non-analitical with respect to the order parameter, contrarily the conventional TDGL type, the resulting equations still allow for a numerical modeling of transient processes related to space- and spatiotemporal vorticity in DWs.
\end{abstract}




\section{Introduction.}

\subsection{Inspirations from I.E. Dzyaloshinskii.}

The authors had a chance to publish together with I.E. Dzyaloshinskii (IED in the following) the article [1] on doubly-quasi-periodic solitonic lattices emerging in a 1D electronic system under simultaneous effects of the charge doping away from the half band filling and of the spin polarization. This publication was in the course of our studies in theory of charge density waves (CDW) which had been started in 1976 by one of us (SB, a thankful disciple of IED) under the inspiration and initially with participation [2, 3] of IED. The trick of the chiral transformations having been emploied in [2] (see below in Sec.3.2), actually suggested by IED, provided a handy frame to study adiabatic models of CDWs which later would lead SB to notice an instability of normal electrons or holes, excited or injected to a CDW, towards formation of solitons with the electron buried at the solitons' midgap state, see [4] for a review. The resulting physics of microscopic solitons and their arrays [5] can be traced back to IED invention of the commensurability locking (published neglectfully only in conference proceedings [6]) and forth to his work on exact solutions of many-body lattice models [7, 8, 9, 10]. Much later, an indirect inspiration came to us [11, 12 from the article [13] where IED had noticed that a presence of a dislocation in a crystal possessing the antiferromagnetic spin order enforces a curious half-integer vortex of spins' rotations.

In spite of an antiquity and following periods of intensive developments, these old studies have left some intriguing openings and unresolved problems which we succeeded to formulate only later in [11, 12, 14] and to detail very recently in [15, 16]. In this short review we shall address some of these issues. First, we shall describe combined topological defects - half-integer vortices of displacements and spin rotations in incommensurate SDWs which might necessarily appear under applications of the driving electric field. These considerations are related to IED work [13] and are coherent with a persistent interest in fractional vortices: from Helium A $([17,18$, about the same time as the IED work) and triplet superconductors (reviews [19, 20]) to FFLO phase [21, 22] and Bose-condensate of polaritons [23], see also [24] for a more recent review of the literature taking into account also experimental attempts.

Our results on SDWs have been published only briefly in conference proceedings [11, 12]; here we shall update them, particularly adding new results on a numerical modelling of static vortices-dislocations and nonstationary phase-slip processes ( $1+1$ space-time vortices). Next we shall quote and augment for modelling quite a recent development [15] on construction of the chirally invariant (recall [2]) description of transient processes in a CDW 
or a SDW in presence of normal carriers. This formulation is free from a drawback of the non-conservation of condensed particles which we show to be inherent to the traditional TDGL approach. Beyond the frame of this article, recall also the most recent work [16] (devoted to ours top teacher I.M. Khalatnikov) on a hydrodynamics of a DW at presence of an ensemble of intrinsic defects - phase solitons or dislocation loops. This direction is in line with long standing interests of IED in topological defects, recall [25] and particularly [26].

\subsection{Significance of static and transient topological con- figurations in incommensurate sliding density waves.}

Embedded or transient topologically nontrivial configurations are common among symmetry broken ground states of electronic systems. Among them, beyond vortices and the FFLO state in superconductors, there is a vast family of electronic crystals, including charge density waves (CDWs), spin density waves (SDWs), Wigner crystals and stripes arrays; with CDWs, and also SDWs, presenting the most popular and convenient object of studies (see reviews [27, 28, 29, 30]).

A unique property of electronic crystals is related to their ability for the collective current conduction by means of sliding. It is ultimately related to appearance, under stresses from the electric field or imbalances of normal particles, of topologically nontrivial objects like solitons, vortices, dislocations, and transient processes of phase slips; all that gives rise to a rich complex of nonlinear and nonstationary behavior. We refer to recent publications [16, 31] for a literature review concerning modern experimental manifestations and various theoretical contributions.

The ground state degeneracy allows for configurations connecting equivalent while different states across a disturbed area. These configurations are protected either topologically or by conservation laws of the charge or the spin. They are known commonly under a generic name of "topological defects" which includes extended objects like planes of domain walls as arrays of solitons [4, 32], lines or loops of dislocations [33, 34, 35] as phase vortices, and local objects like phase and amplitude solitons [4, 5, 36].

The density waves (DWs) are seen as superstructures, usually weak $A \ll 1$ and hence harmonic $\sim A \cos \left[\vec{q}_{0} \vec{r}+\varphi(\vec{r})\right]$, produced by modulations of electronic charges and atomic displacements or of electronic spins, for CDWs or SDWs correspondingly. In most common quasi-1D systems the density wave (DW) is a kind of an elastic uniaxial crystal, which displacements are given by distortions of the DW phase $\varphi(\vec{r})$. Actually, phase displacements 
can be unlimitedly large which brings about such spectacular phenomena as the gigantic permittivity (in this respect the DW can be interpreted as a ferroelectric sitting always exactly at the transition temperature) and even more striking effect of the collective Froehlich conductivity due to the overall sliding with the collective current proportional to the phase velocity $j_{c} \propto \partial_{t} \varphi$. The DW sliding is ultimately related to the current conversion process which necessarily involves topological defects like solitons, phase slips, dislocation lines/loops. The conversion of the normal current to the collective one passes through consecutive dynamic and kinetic steps: free electrons injected from the contact transform dynamically to amplitude solitons (see for review [4, 36]) which keep carrying the electron's spin $1 / 2$ while the local charge is compensated to zero. Pairs of amplitude solitons merge into spinless $2 \pi$ phase solitons of the even lower energy, thus forming local microscopic phase slips. Their subsequent aggregation forms edge dislocation lines (D-lines) or loops (D-loops) expanding across the sample, thus completing the conversion of excess concentration of normal carriers to new periods of the DW superstructure. Macroscopically, the phase slip develops as the edge D-line proliferating/expanding across the sample [37, 38] or as the plane vanishing of the DW amplitude across a narrow sample [39, 40, 41, 42]. Beyond phase slips, the dislocations are supposed to participate in depinning via creation of metastable deformations in the course of the DW sliding over the host lattice imperfections [43, 44, in the "Narrow Band Noise" generation, and also arise in contact structures. The point defects $( \pm 2 \pi$-solitons as "add-atoms" and "vacancies" or also as nucleus dislocation loops) compete with electrons as normal carriers, also providing a material for the climb of dislocations required for their expansion.

Properties of dislocations are strongly affected by Coulomb forces and hence depend on screening facilities of free carriers. The DW deformations generate a local charge density $n_{c} \propto \partial_{x} \varphi$ which brings about a high cost of the Coulomb energy. The Coulomb enhancement of the dislocation energy plays an intriguing role in Spin Density Waves bringing to life a kind of a mixed topological object: a half - integer dislocation combined with a semi vortex of a staggered magnetization vector. The phase slips, necessary for the current conversion or the depinning, should proceed via propulsion of these combined objects which provides a natural interpretation for a confusing 2fold enhancement of a frequency generated by moving Spin Density Waves in comparison with Charge Density Waves.

Beyond transient dislocations contributing to phase slips, static arrays of dislocations can appear in lateral geometries when the electric field is applied transversely to the direction $x$ of the CDW sliding. Indeed, the interaction energy $e \Phi \partial_{x} \varphi / \pi$ of the deformed CDW with the electric potential $\Phi$ resem- 
bles the one for a superconductor under a magnetic field described by the vector potential with the component $A_{x} \equiv \Phi$. Then the transverse, to the chains, electric field $E_{y}=-\partial_{y} \Phi$ acts upon the CDW phase analogousely to the action of the transverse magnetic field in a superconducting film. Hence an array of static dislocations should appear above the electric field threshold as the vortex lattice above $H_{c 1}$ in a superconductor. Nevertheless the situation in a CDW is more complicated technically because of a strong intrinsic field generated by D-lines which requires for a self-consistent treatment contrary to the common practice in superconductors were the magnetic field is commonly treated as the external one. The physics of vortices in CDWs is also more complex because of the interaction of the CDW phase also with the concentrtion $n$ of normal carriers via the energy $n \partial_{x} \varphi \hbar v_{F} / 2$ describing the breathing of the Fermi level following the CDW deformations.

Figures 1 and 2 illustrate the sequences of phase slips and the static dislocation. The plots were obtained from numerical modelling of a TDGL type equations as it has been described elsewere [15, 45, 46].

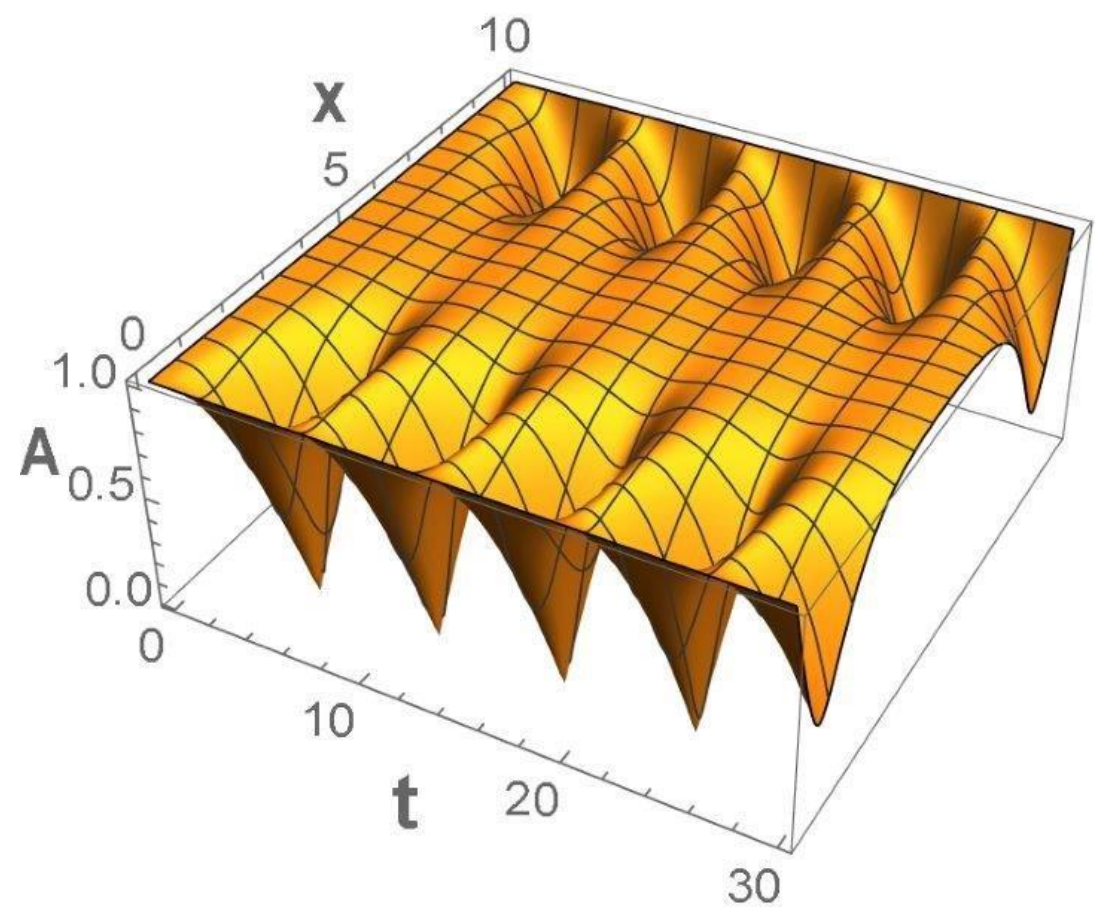

Figure 1: A static array of dislocations appearing in the electric field applied transversely to the direction $x$ of DW displacements. 


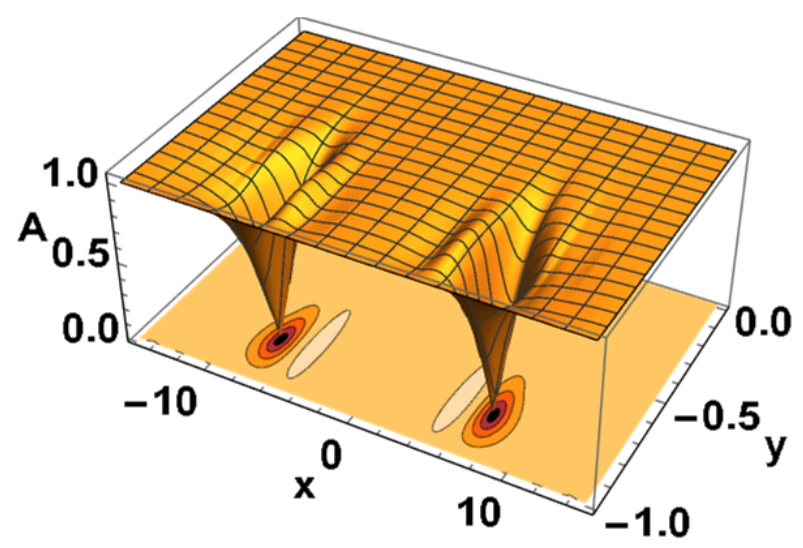

Figure 2: A static array of dislocations appearing in the electric field applied transversely to the direction $x$ of DW displacements.

\section{Combined half-integer dislocation and the magnetization vortex in a spin density wave.}

CDW and SDW are characterized by scalar and vector order parameters: $\eta_{c d w}(\vec{R})=A \exp (i \varphi)$ and $\vec{\eta}_{s d w}=A \vec{m} \exp (i \varphi)$, where $\vec{m}$ is the unit vector of the staggered magnetization and $\vec{R}=\left(x, \vec{r}_{\perp}\right)$ with $x$ being the chain direction,. Here we will show that SDWs allow for, and actually prefer, an unusual $\pi$ phase vortex which is forbidden in CDWs where only $2 \pi$ vortices are allowed [11, 12]. Namely, in SDW conventional dislocations loose their priority in favor of complex topological objects: a half-integer dislocation combined with a semi-vortex of the staggered magnetization vector, as illustrated in Figure 3.

A possible manifestation of half-integer vortices can be found in the NBN generation originated by a periodical sequence of phase slips in the course of sliding. The $\pi$ - phase slip reduces twice the universal ratio $\Omega / j$ of the fundamental NBN frequency $\Omega$ to the mean sliding current $j$ as we shall describe in details below. The splitting of the normal $2 \pi$-dislocation to the $\pi$ ones is energetically favorable due to Coulomb interactions which harden the elasticity with respect to the phase displacements while leaving intact the rigidity with respect to spin rotations. The magnetic anisotropy confines half-integer vortices in pairs connected by a magnetic domain wall which binding can be overcome by the Coulomb repulsion. The energy density of spin rotations $\vec{m}(\vec{R})$ in SDWs is not affected by Coulomb forces:

$$
W_{\text {spin }}\{\vec{m}\}=\frac{1}{2 N_{F}}\left[\tilde{C}_{\|}\left(\partial_{x} \vec{m}\right)^{2}+\tilde{C}_{\perp}\left(\left(\partial_{y} \vec{m}\right)^{2}+\left(\partial_{z} \vec{m}\right)^{2}\right)+W_{s a}\right]
$$




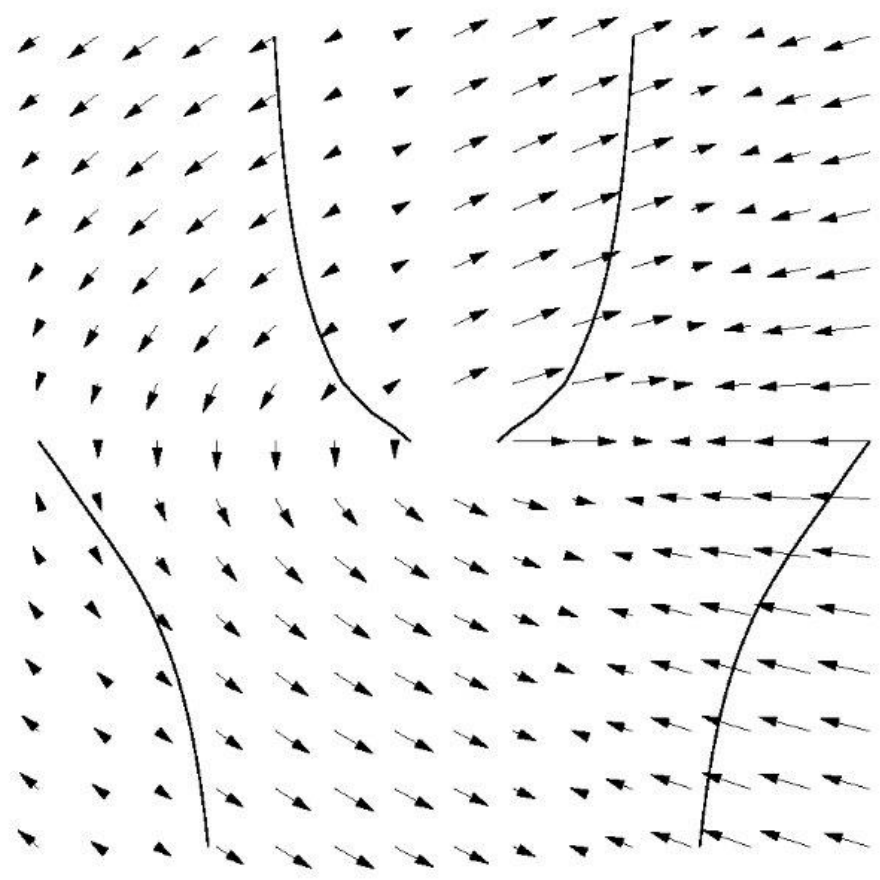

Figure 3: Vector-field $\vec{m}$ for the half-integer dislocation combined with the semi-vortex of the magnetization. Solid lines indicate constant DW phases. Due to the presence of the half-integer dislocation, the number of sites changes between the upper and the lower rows from 2.5 periods $(6+6+3)$ to 2 periods $(7+5)$.

Here $\tilde{C}_{\|}, \tilde{C}_{\perp}$ are the elastic moduli related to the rotation of the staggered magnetization unit vector $\vec{m}, W_{s a}$ is the spin anisotropy energy. $\tilde{C}_{\|}, \tilde{C}_{\perp}$ are similar to bare moduli of phase displacements taken without Coulomb interactions: $C_{\|}^{0}, C_{\perp}$ below in Eq. (2).

The energy for deformations related to phase displacements in both CDW and SDW takes a form

$$
W_{c h r g}\{\varphi\}=\frac{1}{2 N_{F}}\left[C_{\|}^{0}\left(\partial_{x} \varphi / \pi\right)^{2}+C_{\perp}\left(\nabla_{\perp} \varphi / \pi\right)^{2}\right]+W_{C}+W_{s t r},
$$

where $N_{F}=2 /\left(\pi \hbar v_{F}\right)$ and $v_{F}$ are the density of states and the Fermi velocity of the parent metal. Dimensionless parameters $C_{\|}^{0}$ and $C_{\perp}$ are the normalized compression and share moduli; $C_{\perp} \sim\left(A T_{c} N_{F} a_{\perp}\right)^{2}$ is a measure of the interchain coupling related to the transition temperature $T_{c} . W_{\text {str }}$ is the stress energy from an applied electric potential and/or from disbalance of normal carriers which promote deformation and/or the motion of the DW, see more in Sec.3. 
The Coulomb part of the energy $W_{C}$ comes from the local charge density related to the DW displacements: $n_{c}=e \rho_{c} \partial_{x} \varphi / \pi s$, where $\rho_{c}$ and $\rho_{n}=1-\rho_{c}$ are the normalized densities of the condensate and of the normal carriers. The Coulomb interactions drastically affect the charged phase deformations of dislocations greatly increasing their energy and stretching the shape in the chains' $x$ direction [34] as we shall remind below with some more details in the Appendix. Vaguely, the combined effect of Coulomb interactions and the screening results in hardening of the effective compessibility [47] which vanishes at the transition and diverges at low $T$ with freezing out of normal carriers. The effective compressibility $C_{\|}$hardens with growing $r_{\perp}$ (starting from $C_{\|}^{0}$ at shortest interchain distances) as $C_{\|} \sim r^{2} / r_{0}^{2}$ beyond the screening length of the parent metal $r_{\perp}>r_{0} \sim 1 \AA$, until it saturates beyond the screening length $r_{s c r}=r_{0} / \sqrt{\rho_{n}}$ at the value which grows activationally with lowering $T$. At $r_{\perp}>r_{s c r}$

$$
C_{\|}^{0} \Rightarrow C_{\|}=\rho_{c} / \rho_{n}: C_{\|} \propto \rho_{c} \propto A^{2} \propto\left(T_{c}-T\right) \text { at } T \rightarrow T_{c}
$$

and

$$
C_{\|} \propto \rho_{n}^{-1} \propto \exp (\Delta / T) \text { at } T \rightarrow 0
$$

The resulting big energy of dislocations at small $\rho_{n}$ does not prevent their creation which just requires for bigger applied potentials, but the Coulomb energy changes drastically the energy dependence of the dislocation on its position $Y$ (with respect to a counterpart or a surface). While the spinvortex energy per its unit length is a standard $W_{V} \sim T_{c} \ln \left(Y / a_{\perp}\right)$, for the dislocation as the charged phase vortex this form of the energy is reached only when the Coulomb interaction is screened at $Y \gg r_{s c r}$ where $W_{D} \sim$ $T_{c}\left(r_{s c r} / r_{0}\right) \ln \left(Y / r_{s c r}\right)$ with the energy scale being greatly enhanced as $r_{s c r} / r_{0}$.

Coulomb interactions become even more important in an intermediate (wide at low $T$ ) region $r_{0}<r_{\perp}<r_{s c r}$ governed by the nonlocal, due to unsceened Coulomb interactions, elasticity with $C_{\|} \sim r^{2} / r_{0}^{2}$. For dislocations with $r_{s c r} \gg Y \gg r_{0}$ a curious confinement law is established with $W_{D} \sim$ $T_{c} Y / r_{0}$ meaning a constant force acting upon the D-line.

The energies $W_{V}(Y)$ and $W_{D}(Y)$ are similar only near $T_{c}$ at small $A$, hence the vanishing gap $\Delta$, when the abundant free carriers screen Coulomb interactions already at shortest distances. Otherwise, at the developed gap $\Delta>T$, the energy of dislocations is greatly enhances with respect to that of vortices which brings about the option of their spitting into combined half-integer vortices. In SDWs the Coulomb enhancement of the dislocation energy plays a principal role bringing to life a special combined topological object: the half-integer dislocation $\varphi \rightarrow \varphi+\pi$ accompanied by the $180^{\circ}$ rotation $\mathcal{O}_{\pi}$ of the staggered magnetization $\vec{m} \rightarrow-\vec{m}$. Indeed, the SDW 
order parameter $\vec{\eta}=\vec{m} \cos (Q x+\varphi)$ allows for the following three types of self-mapping $\vec{\eta} \rightarrow \vec{\eta}$ associated with vorticities $\nu_{\phi}$ and $\nu_{m}$.

i. normal dislocation $D_{2 \pi}: \varphi \rightarrow \varphi+2 \pi \equiv \varphi$ and $\vec{m} \rightarrow \vec{m}, \nu_{\phi}=1$ and $\nu_{m}=0$; ii. normal $\vec{m}$ - vortex $V_{2 \pi}: \vec{m} \rightarrow \mathcal{O}_{2 \pi} \vec{m} \equiv \vec{m}$ and $\varphi \rightarrow \varphi, \nu_{\phi}=0$ and $\nu_{m}=1$; iii. combined object $D_{\pi} V_{\pi}: \varphi \rightarrow \varphi \pm \pi$ and $\vec{m} \rightarrow \mathcal{O}_{\pi} \vec{m}=-\vec{m}, \nu_{\phi}= \pm 1 / 2$ and $\nu_{m}= \pm 1 / 2$.

In the last case both the orientational factor $\vec{m}$ and the translational one $\cos (Q x+\varphi)$ change the sign, but their product in $\vec{\eta}$ stays invariant, as it is demonstrated schematically in Figure 3. Remind that at a given position the energies of vortices depend on their winding numbers as $\propto \nu_{\varphi, m}^{2}$

We must compare the energies of objects i. and iii. under the requirement of the charge conservation i.e. preserving the total phase vorticity $\sum \nu_{\phi}$. For magnetic vortices the total vorticity $\sum \nu_{m}$ does not need to be conserved but in the bulk it must be kept zero; otherwise the energy of noncompensated vortices diverges logarithmically at large distances. For phase dislocations the energy divergence is not a limitation since it is compensated by driving potentials. Hence, the only decomposition path for the conventional dislocation of the case i. to the two pairs of half-vortices of the case iii. is $D_{2 \pi} \Rightarrow\left\{D_{\pi}, V_{\pi}\right\}+\left\{D_{\pi}, V_{-\pi}\right\}$. If the energy parameters for both $D$ and $V$ were the same, then the dissociation cost is zero: $\nu_{2 \pi}^{2}=1 \Rightarrow 4 \nu_{\pi}^{2}=1$ and the result will depend on a tiny balance of similar coefficients. But with dominating Coulomb energy of dislocations, as expected at low $T$, the magnetic vortex energy can be neglected, then the decomposition gains nearly half of the energy $\left(\nu_{2 \pi}=1\right)^{2} \Rightarrow 2\left(\nu_{\pi}=1 / 2\right)^{2}=1 / 2$ which makes it inevitable.

Figures $4, \mathrm{a}, \mathrm{b}$ demonstrate results of our modeling of the evolution from a single phase-only integer vortex - the dislocation - to the split pair of halfinteger combined vortices. Figure 4 a presents the 3D plot for the SDW order parameter amplitude; Figure $4 \mathrm{~b}$ presents vector fields of the CDW phase and of the spin rotation on the background of the density plot for the amplitude.

Figure 4.

The spin anisotropy of an "easy plane" type allows for a free rotation of spins, so it will not affect the above conclusions. The same will hold in the pure "easy axis" case at presence of a magnetic field $H>H_{s f}$ exceeding the spin-flop field $H_{s f} \sim 1 T$ above which the spins will be tilted, thus possessing a free rotation at the hard plane. The known SDW crystals possess low symmetries which originates the spin anisotropy in all three directions. Being small, the anisotropy will not affect the arrangement in a vicinity of the DL but at large distances from the DL the $\pi$-rotation of spins will be concentrated in space within the Neél domain wall, as it has been outlined already in [13]. The wall will form a string (a plane in 3D) which tempts to confine the two combined objects. 

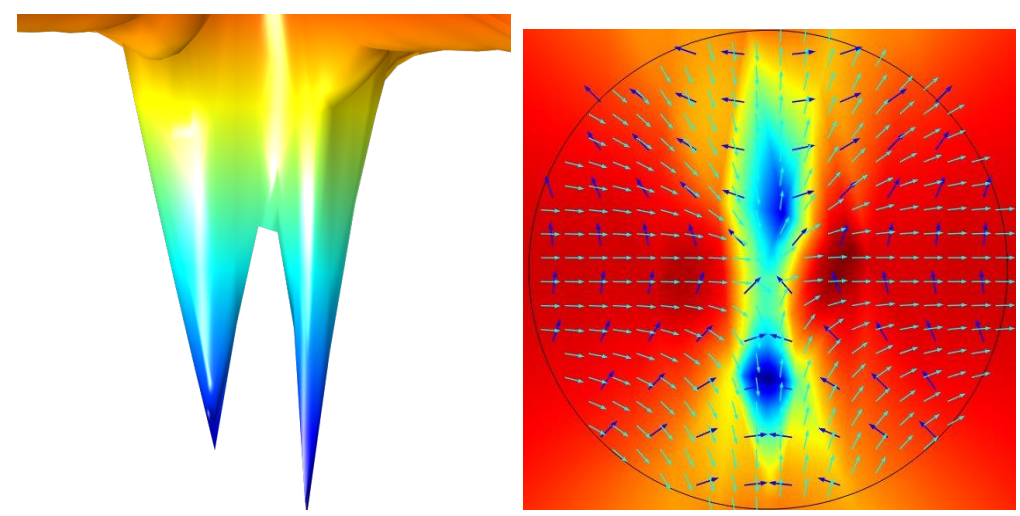

Figure 4: Results of the modeling of the evolution from a single integer dislocation to the split pair of half-integer combined vortices: the order parameter amplitude $A(x, y)$ (left panel); vector fields of the CDW phase and of the spin rotation on the background of the density plot for the amplitude (right panel).

Unusually, there will be no confinement within a quite wide region $r<r_{s c r}$ where the linear law, rather than conventional logarithmic one, for the Dline energy takes place as we have sketched above and in the Appendix. Here the total energy gain with respect to the normal DL is $-E_{C} N / 2$ where $E_{C} \sim T_{c} a_{\perp} / r_{0}$ and $N=Y / a_{\perp}$ is the number of chains separating combined vortices. This repulsive anti-confinement energy directly overruns the energy lost due to domain wall formation $W_{\text {spin }}^{A}=w^{A} N$, both having the similar $N$ dependence. Usually $w^{A} \sim 1 \mathrm{~K} /$ chain $\ll E_{C} \sim 10^{1} \mathrm{~K}$, so the net interaction between the two semivortices is strongly repulsive.

Beyond the screening length $r>r_{s c r}$, the Coulomb energy slows down while the $W_{\text {spin }}^{A}$ keeps growing linearly, then the total energy gain of two objects with respect to one DL is

$$
W=-E_{0} \ln N+w^{A} N, E_{0} \sim T_{c}\left(r_{s c r} / r_{0}\right)=T_{c} / \sqrt{\rho_{n}}
$$

Hence there is an equilibrium distance between the semi-vortices $N_{e q} \sim$ $\left.E_{0} / w^{A} \propto 1 / \sqrt{\rho_{n}}\right)$ which diverges with freezing out of the screening when $\rho_{n} \rightarrow 0$. Already at accessibly low temperatures, the string length may reach the sample width which is typically $\sim 1 \mu \mathrm{m}$.

\subsection{Combined topological defects and the NBN gen- eration.}

A remarkable phenomena of sliding DWs is the generation of the so called Narrow Band Noise (NBN) [28] which is a coherent periodic unharmonic 
signal with the fundamental frequency $f$ being proportional to the mean $d c$ sliding current $j$ (per chain) yielding the universal ratio of $e f / j$. In CDWs ideally ef $/ j=1 / 2$ which corresponds to carrying of two electrons by displacing of the CDW by its wave length $\lambda$. In SDWs the ratio has been accessed only indirectly [48, 49] with different experiments being in favor of either $e f / j=1 / 2$ or the twice higher ratio $e f / j=1$. Surprisingly, there is no consensus on the origin of such a bright and widely exploited effect as the NBN, with two main pictures competing.

i. The Phase Slip Generation (PSG) model suggests that the NBN is generated by phase discontinuities of phase slip processes occurring periodically near injecting junctions [37, 38, 39, 40, 41, 42] (and references to later experiments in [50]).

ii. The earlier and better known Wash-Board Frequency (WBF) model suggests that the NBN is generated extrinsically in the course of the DW passing through the host lattice sites or its defects [28, 51]. Recall that the oscillating densities of the charge or of the lattice displacements in the CDW and the SDW are different:

$$
\rho_{c d w} \sim \eta_{c d w}=A \cos [Q x+\varphi] \text { but } \rho_{s d w} \sim \delta\left(\vec{\eta}_{c d w}\right)^{2} \Rightarrow A^{2} \cos [2 Q x+2 \varphi]
$$

- the charge modulation wave length in the SDW is only half of the CDW one $\lambda=2 \pi / Q$. Hence within the WBF model the NBN frequency is doubled for the SDW.

The observation of the twice different ratios was considered to be in favor of the WBF model which nevertheless has an unresolved weaknesses. The original concept implied the interaction between the rigid DW and the regular host lattice $\sim \cos (n \varphi)$ where the commensurability index is typically $n=4$ which would give an $n$ - fold WBF contrary to experiments. A later belief was that the necessary potential $V_{i m p}$ is provided by the host impurities. But actually $V_{i m p} \sim \cos \left(Q x_{i}+\varphi\right)$ so that the positionally random phase shifts $-Q x_{i}$ will prevent any coherence in the linear response.

An intermediate scenario suggests that the DW does not slide at the sample side surface so that the coupling $\sim \cos \left(\varphi_{\text {bulk }}-\varphi_{\text {surface }}\right)$ with $\varphi_{\text {bulk }} \propto t$ and $\varphi_{\text {surface }}=$ cnst would provide a necessary WBF. This separation of moving and staying layers requires for a sequence of dislocations thus building a bridge to a version of the PSG scenario. The PSG model is attractive because the PSs are ultimately necessary already to provide the current conversion at the contacts. A weak point of the PSG model is to explain their regularity as shown by a remarkably high coherence of the NBN in experiments. 


\section{Two-fluid hydrodynamics for collective and normal variables in DWs.}

\subsection{Problems with Ginzburg -Landau like models and their time-dependent generalizations for density waves.}

Describing and modeling inhomogeneous states and transient processes in DWs calls for an efficient phenomenological theory which should take into account the degenerate order parameter, normal carriers and the electric field. Related cases of vortices in superconductors and dislocations in density waves [39, 40, 41] are described usually within Ginzburg-Landau (GL) like models and their time-dependent (TDGL) generalizations. The essence of these approaches is that electrons are excluded ("fermions are integrated out") at the earliest stage in favor of the order parameter. While being consistent for the explicitly gauge-invariant theory of superconductors [52, 53], this approach fails for density waves and other electronic crystals. The discrepancy is particularly pronounced for processes with a dynamical vorticity - phase slips or moving dislocations, leading to a non-physical production or elimination of condensed particles in their cores. Working at all steps explicitly with interfering order parameter and normal electrons, employing the chiral invariance, and taking the account for the related quantum anomaly have allowed us [15] to construct the treatable theory without descending to the burdens of microscopic calculations. The price paid is that the theory is non-analytical with respect to the order parameter which originates some particular difficulties in numerical modeling. Nevertheless, with some simplifications we succeed to demonstrate the dynamical creation of vortices and phase slips processes, including pictures presented in this article.

In the TDGL theory for superconductors, the charge conservation is automatically preserved because the equation of motion for the order parameter contains only gauge invariant combinations of the phase and the electromagnetic field. But in CDWs the order parameter phase characterizes breaking of the chiral (translational) rather than the gauge symmetry. The equations of motion for the CDW phase certify the local equilibrium of forces (elastic, Coulomb, frictional) with no relation to the charge conservation. Instead, the last is usually preserved automatically by construction of the charge and the current densities:

$$
n_{c}=\rho_{c} \partial_{x} \varphi / \pi, j_{c}=-\rho_{c} \partial_{t} \varphi / \pi
$$

(to be compared with $j_{S C} \propto \rho_{s c} \partial_{x} \vartheta$ for the current dependence on the phase 
$\vartheta$ in superconductors). (Here and below the currents refer to the number of particles and the electronic charge $e$ is incorporated into the corresponding potentials.) The normalized (to $T=0$ ) condensate density or the phase rigidity $\rho_{c}(A)$ has a property that $\rho_{c}(0)=1$ and $\rho_{c}(A) \sim A^{2} \rightarrow 0$ at $A \rightarrow 0$. The expressions (3) can ensure the charge conservation automatically indeed, but only if the CDW amplitude is invariable: $A(t, x)=$ cnst, otherwise

$$
\frac{d n_{c}}{d t}=\partial_{t} n_{c}+\partial_{x} j_{c}=\frac{1}{\pi}\left(\partial_{x} \rho_{c} \partial_{t} \varphi-\partial_{t} \rho_{c} \partial_{x} \varphi\right) \neq 0
$$

The charge conservation $d n_{c} / d t=0$ is violated in the current-carrying state $\partial_{t} \varphi \neq 0$ if $\rho_{c}$ is not space homogeneous $\partial_{x} \rho_{c} \neq 0$ and in the strained (charged) state $\partial_{x} \varphi \neq 0$ if $\rho_{c}$ varies in time $\partial_{t} \rho_{c} \neq 0$. The effect is particularly disturbing in description of motion or nucleation of vortices or of phase-slip processes when space and time derivatives are high near the vortex core or the amplitude node. It looks that for a general spacio-temporal regime there is no explicit way to define the CDW collective charge and current via the order parameter alone. It is ultimately necessary to take into account the normal carriers explicitly, without integrating them out prematurely. At first sight, that would require for descending to the fully microscopic theory with its notorious complications even in linear or gapless regimes [40, 54]. Still, there is a way [15] to keep the phenomenology which is based on the knowledge of chiral invariance and transformation, importantly taking into account the chiral anomaly.

The paradox is present also in theory of an arbitrary electronic crystal beyond the quasi one-dimensionality and the particular microscopics inherent to DWs. E.g. for a Wigner crystal the charge and the current densities are expressed via the field of displacements $\mathbf{u}(\mathbf{r}, t)$ (which generalizes $\mathbf{u} \rightarrow \varphi \hat{\mathbf{x}}$ of DWs) as $n_{c}=-\nu(\nabla \cdot \mathbf{u}), \mathbf{j}_{c}=\nu \partial_{t} \mathbf{u}$ where $\nu(\mathbf{r}, t)$ is the filling factor of the crystal unit cell by electrons. Then the conservation law is violated for a variable $\nu(x, t)$ :

$$
\frac{d n_{c}}{d t}=\partial_{t} n_{c}+\left(\nabla \cdot \mathbf{j}_{c}\right)=\left(\nabla \nu \cdot \partial_{t} \mathbf{u}\right)-\partial_{t} \nu(\nabla \cdot \mathbf{u}) \neq 0
$$

\subsection{The tool of the chiral transformation and of the related anomaly.}

The Lagrangian $H-i \hbar \partial_{t}$ for electrons in 1D CDW has a form:

$$
\mathcal{L}=\left(\begin{array}{cc}
-i \hbar \partial_{t}-i \hbar v_{F} \partial_{x}+\Phi-v_{F} A_{x} & \Delta e^{i \varphi} \\
\Delta e^{-i \varphi} & -i \hbar \partial_{t}+i \hbar v_{F} \partial_{x}+\Phi+v_{F} A_{x}
\end{array}\right)
$$


where $\Phi$ and $A_{x}$ are the scalar and the vector potentials. The chiral transformation $\psi_{ \pm} \longrightarrow \psi_{ \pm} \exp ( \pm i \varphi / 2)$ [2], brings the wave function to the local frame of a distorted CDW phase. It eliminates the phase factors in non-diagonal elements in $\mathcal{L}$, but in expense of additional parts in $\Phi$ and $A_{x}$ :

$$
\begin{gathered}
\Phi \longrightarrow V=\Phi+\hbar v_{F} / 2 \partial_{x} \varphi, A_{x} \longrightarrow A_{x}^{*}=A_{x}+\hbar /\left(2 v_{F}\right) \partial_{t} \varphi \\
E_{x} \longrightarrow E_{x}^{*}=-\partial_{x} V+\partial_{t} A_{x}^{*}=E_{x}-\hbar v_{F} / 2\left(\partial_{x}^{2}-\partial_{t}^{2} / v_{F}^{2}\right) \varphi
\end{gathered}
$$

The additions to $\Phi$ and $A_{x}$ from $x, t$ derivatives of the phase are naturally interpreted as the Fermi energy shift $\delta E_{F}=v_{F} \delta P_{F}$ following the Fermi momentum shift $\delta P_{F}=\hbar \partial_{x} \varphi / 2$ under the CDW phase deformations. Under the CDW phase deformation and the applied electric field $E_{x}$, the electrons experience the chiral invariant potentials $V$ and $A_{x}^{*}$ and the longitudinal force $E_{x}^{*}$.

At first sight, we have arrived at the transparent picture of a 1D Dirac semiconductor with the gap $2 \Delta=2 A \Delta_{0}$ under the effective electric field (7), and it looks straightforward to exclude the fermions to arrive at an effective action $S\{\Phi, \varphi, A\}=\int W d x d t$. And here we arrive at puzzling contradictions:

i. In view of Eq.(7), the free energy $W$ should contain the potential and the phase only in the invariant combination $V$. Having choosen the phase as $\left(\hbar v_{F} / 2\right) \partial_{x} \varphi=-\Phi+$, the effective potential $V$ disappears from the action, then no density and no polarization are perturbed with respect to $\Phi$ which contradicts to basic properties of the CDW as both the semiconductor with respect to electrons and the metal in the collective behavior.

ii. We definitely expect $W$ to contain the term $\propto \rho_{c}\left(\partial_{x} \varphi\right)^{2}$ where $\rho_{c}$ is a collective density responsible for the phase rigidity. But treating the Lagrangian (5) perturbatively with respect to the effective electric field (7) we evidently should get

$$
\delta W_{n}=-\frac{\varepsilon(k, \omega)}{8 \pi e^{2}}\left(E_{x}^{*}\right)^{2}=-\frac{\varepsilon(k, \omega}{8 \pi e^{2}}\left(-\frac{\hbar v_{F}}{2} \frac{\partial^{2} \vartheta}{\partial x^{2}}+E_{x}\right)^{2}
$$

where $\epsilon$ is the dielectric susceptibility as a function of the wave number $k$ and the frequency $\omega$ (the last will be neglected here for shortness). At small $k$ :

$$
\epsilon=\epsilon_{\Delta}+\frac{1}{(l k)^{2}}, \frac{1}{l^{2}}=\frac{\rho_{n}}{r_{0}^{2}}, \rho_{n}=\frac{d n}{d \zeta} N_{F}^{-1}
$$

Here $r_{0}$ is the Thomas-Fermi radius of the parent metal, $n$ is the concentration of free electrons related to their chemical potential $\zeta$, and $\rho_{n}$ is the normalized DOS which also will be found to be the normal density. The dielectric constant $\epsilon_{\Delta} \propto\left(r_{0} \xi_{0}\right)^{-2}$ collects the polarization of electrons gapped 
by the CDW while $\rho_{n}$ comes from conducting carriers thermally excited or injected [55] above the gap providing the finite screening length $l=r_{0} / \sqrt{ } \rho_{n}$. Both contributions to $\delta W_{n}$ bring drastic contradictions:

a) The term with $\epsilon_{\Delta}$ in Eq. (9) yields to (8) the forth order gradients of the phase $\left(\partial_{x x}^{2} \varphi\right)^{2}$ instead of the expected second order one $\propto\left(\partial_{x} \varphi\right)^{2}$.

b) The singularity in $k$ in the term with $\rho_{n}$ seems at first to serve fortunately by canceling excess gradients leading to the contribution $\propto\left(\partial_{x} \varphi\right)^{2}$ :

$\delta W_{\rho n}=-\rho_{n}\left(\hbar v_{F} / 4 \pi\right)\left(\partial_{x} \varphi+\pi N_{F} \Phi\right)^{2}$ which expression brings two confusions with respect to the expected $W_{\rho c}=\rho_{c}\left(\hbar v_{F} / 4 \pi\right)\left(\partial_{x} \varphi\right)^{2}$ - even the signs in expressions for $W_{\rho n}$ and $W_{\rho c}$ are opposite, and also temperature dependencies are conflicting among coefficients $\rho_{n}$ (expected to rise from zero at $T=0$ up to 1 at $T_{c}$ ) and $\rho_{c}$ (expected to fall to zero at $T_{c}$ starting from 1 at $T=0)$.

These contradictions can be traced back to the notion of the chiral anomaly and they can be cured by properly taking this anomaly into account. The chiral anomaly is ubiquitous to the premature linearization of electronic spectra. First, in the course of the linearization, the control is lost of the position of the bottom of the electronic band, hence of the difference between actions of the external potential $\Phi$ and of the Fermi energy shifting $\delta E_{F}$ which wrongly appear to be additive. Moreover, the whole energy cost of the chiral transformation (CT) $\delta W_{C T}$ is lost. This energy can be captured from the non-linearized spectrum of the normal metal if we consider the chiral transformation perturbation $\delta n_{C T}=\partial_{x} \varphi / \pi$ as a redistribution of the total particle density accompanying the this transformation.

$$
\delta W_{C T}=\frac{\hbar v_{F}}{4 \pi}\left(\partial_{x} \varphi\right)^{2}+\frac{\Phi}{\pi} \partial_{x} \varphi
$$

where the first term is the density perturbation cost $\left(\delta n_{C T}\right)^{2} /\left(2 N_{F}\right)$ and the second term is its potential energy $\delta n_{c} \Phi$. Beyond these physical arguments [56, 57], the derivation of the chiral anomaly in the spirit of the field-theory procedure of regularization of fermionic determinants was demonstrated for CDWs [58] and for SDWs [58, 59], recall also the special field-induced SDWs [60]. In the Appendix we shall briefly clarify the microscopic origin of the chiral anomaly paradox.

Bringing together the nonperturbative contribution 10 and the perturbative one (8), we get

$$
W_{t o t}=\delta W_{\rho n}+\delta W_{C T}=\rho_{c}\left(\frac{\hbar v_{F}}{4 \pi}\left(\partial_{x} \varphi\right)^{2}+\frac{\Phi}{\pi} \partial_{x} \varphi\right)-\rho_{n} \frac{(\Phi)^{2}}{\pi \hbar v_{F}}
$$

where $\rho_{c}=1-\rho_{n}$. The above equation correctly manifests the expected dependencies in $T$ and $A$ yielding also the important relation $\rho_{c}+\rho_{n}=1$. 
The total charge density becomes

$$
n_{t o t}=\frac{\partial W_{t o t}}{\partial \Phi}=\frac{1}{\pi} \rho_{c} \partial_{x} \varphi-\rho_{n} \Phi N_{F}=\frac{1}{\pi} \partial_{x} \varphi-\rho_{n} V N_{F}
$$

Here the first form of $n_{\text {tot }}$ interpret the CDW charge as $\rho_{c}$ leaving the electronic density to react only to $\Phi$ which approach is common and convenient while ambiguous: with a variable $\rho_{c}$ this form leads to violation of the charge conservation as it was discussed in the previous section. The second form of $n_{\text {tot }}$ lets us to understand that the collective charge density is always the nominal one embracing all electrons in the vacuum and excited states, not reduced by the factor $\rho_{c}$; meanwhile the charge density of normal electrons is perturbed by their actual combined potential $V$ rather than by its part $\Phi$. In this picture, the charge and the current are given by independently conserved counterparts as show in Eq. (3).

The above illustrative discussion was valid in lowest bilinear approximation in gradients and potentials and, more restrictively, for the constant amplitude $A$, hence $\rho_{n}, \rho_{c}$. In the next section we shall suggest a general nonlinear scheme necessary for modeling configurations with vortices.

\subsection{General equations for nonlinear regimes.}

The above relations written for the limit $A=c n s t$ and for small deviations of $n_{e}, n_{h}$ can be generalized by extending the energy functional as $\int W d x$ with

$$
\begin{array}{r}
W=\frac{\hbar v_{F}}{4 \pi}\left[\kappa_{x}\left(\partial_{x} A\right)^{2}+\kappa_{\perp}\left(\nabla_{\perp} A\right)^{2}+\kappa_{\perp} A^{2}\left(\nabla_{\perp} \varphi\right)^{2}\right]+ \\
\left\{\frac{\hbar v_{F}}{4 \pi}\left(\partial_{x} \varphi\right)^{2}+\frac{1}{\pi} \Phi \partial_{x} \varphi\right\}+\left(\Phi+\frac{\hbar v_{F}}{2} \partial_{x} \varphi\right) n+F\left(A, n_{e}, n_{h}\right)-\frac{\varepsilon_{\text {host }} s}{8 \pi}(\nabla \Phi)^{2}
\end{array}
$$

Here the parameter $\kappa_{\perp}$ is the CDW share modulus coming from the interchain coupling of CDWs, and the on-chain rigidity of the amplitude $\kappa_{x} \sim 1$ (to be put $\left.\kappa_{x}=1\right) . F\left(A, n_{e}, n_{h}\right)$ is a free energy as a function of the normalized gap value $A=\Delta / \Delta_{0}$ and of the concentration of normal carriers: electrons $n_{e}$ and holes $n_{h}$ with $n=n_{e}-n_{h}$. The equilibrium value $A_{e q}$ is connected with $n_{e}, n_{h}$ via the minimum of $F\left(A, n_{e}, n_{h}\right)$, in such a way that $A_{e q}\left(n_{e}, n_{h}\right)$ vanishes when $n_{e, h}$, or better say their chemical potentials $\pm \zeta$ surpass critical values, hence the metallic phase with $A=0$ is restored. The terms in brackets \{\} are originated by the chiral anomaly of Eq. 10 coming from background deformations of the CDW phase, the next term $\sim n$ comes from the energy of intrinsic electrons in the combined potential $V$. 
Assuming the dissipative regime for both $\varphi$ and $A$, functional derivatives of (13) yield equations for the time evolution and the Poisson equation for $\Phi$ :

$$
\begin{array}{r}
\partial_{x}^{2} A+\kappa_{\perp} \nabla_{\perp}^{2} A+\kappa_{\perp} A\left(\nabla_{\perp} \varphi\right)^{2}-\partial F / \partial A=\gamma_{A} \partial_{t} A \\
\partial_{x}^{2} \varphi+\pi N_{F} \partial_{x} \Phi+\pi \partial_{x} n+\kappa_{\perp} \partial_{y}\left(A^{2} \nabla_{\perp} \varphi\right)=\gamma_{\varphi} \partial_{t} \\
\partial_{x} \varphi / \pi+n=-N_{F} r_{0}^{2} \nabla^{2} \Phi
\end{array}
$$

Here $\gamma_{\varphi}=\gamma A^{2}, \gamma=$ cnst, $\gamma_{A}=$ cnst are the damping coefficients; $\gamma_{\varphi}$ is related to the sliding $\mathrm{CDW}$ conductivity $\sigma_{C D W}$ as $\gamma_{\varphi} \sigma_{C D W}=N_{F} e^{2} / s=$ $1 /\left(4 \pi r_{0}^{2}\right)$.

In spite of a superficial similarity, these eqs. show striking differences with respect to commonly use TDGL ones. Thus, in the first Eq. the conventional term $\kappa_{\perp} A\left(\nabla_{\perp} \varphi\right)^{2}$ does not find its partner with the $x$ derivative of $\varphi$ as if the longitudinal gradient of $\varphi$ does not suppress the amplitude - the phase-slip nodes would not appear then. In the second and the third eqs. the terms in brackets \{\} containing $\partial_{x} \varphi, \partial_{x x} \varphi$, and $\left(\partial_{x} \varphi\right)^{2}$ are not multiplied by $A^{2}$, so the attempt to present them as derivatives of $\Psi$ will bring $\sim 1 / A, \sim 1 / A^{2}$ singularities: contrary to conventional GL-type equations, now Eqs. 14 15 16 are nonanalytic in the order parameter $\eta=A \exp (i \varphi)$ or in other words singular in its amplitude. Nevertheless, there are hidden cancellations allowing to compensate for singularities, even if implicitly, which is vitally important for allowance of space- and spacio-temporal vortices. With additional approximations, below these compensations will be better exposed or proved at least.

Finally, the kinetics of normal carriers can be taken in the quasi-equilibrium diffusive approximation:

$$
\nabla\left(\hat{\sigma}_{n} \nabla \mu\right)=\left(e^{2} / s\right) \partial_{t} n, \mu_{n}=\zeta+\Phi+\partial_{x} \varphi /\left(\pi N_{F}\right)
$$

with the electrochemical potential $\mu_{n}$ and the conductivity tensor $\hat{\sigma}_{n}$.

For the instantaneous reaction of normal carriers in the limit $\sigma_{n} \rightarrow \infty$ requesting for $\mu_{n}=0$, this relation helps to understand the decomposition of the total charge density $n_{t o t}$. Differentiating over $x$ the general expression for $n_{\text {tot }}$ as given in the LHS of Eq. (16) and using $\mu_{n}=0$ we arrive at

$$
\partial_{x} n_{t o t}=-\rho_{n} N_{F} \partial_{x} \Phi+\rho_{c} / \pi \partial_{x}^{2} \varphi
$$

For $\rho_{n}, \rho_{c}=$ cnst this expession can be integracted to the restricted form of Eq. (12). Here the first term corresponds to the conventional reaction (the screening of the 


\subsection{The limits of the local electroneutrality together with the infinite conductivity.}

The general equations can be substantially simplified and clarified in two limits: the infinite conductivity of normal carriers which yields $\mu_{n}=\zeta+\Phi+$ $\partial_{x} \varphi /\left(\pi N_{F}\right)=0$ and the local electroneutrality $r_{0} \rightarrow 0$ which yields $\partial_{x} \varphi+$ $\pi n=0$. Separately the two limits have been considered in [15; here we shall remind only their joint result. The above local relations allow to express $\Phi, \partial_{x} \varphi$ via $\zeta$ (or equivalently via $n$ ) alone: $\Phi=n / N_{F}-\zeta, \partial_{x} \varphi=-\pi n(\zeta, A)$. Then we arrive at two equivalent forms of equations for the phase:

$$
\begin{gathered}
\partial_{x} \Phi+\left(\pi N_{F}\right)^{-1} \nabla_{\perp} A^{2} \nabla_{\perp} \varphi-\gamma_{\varphi} \partial_{t} \varphi=0, \Phi=\left(n / N_{F}-\zeta\right) \\
\frac{\rho_{c}}{\rho_{n}} \partial_{x}^{2} \varphi+\kappa_{\perp} \nabla_{\perp}\left(A^{2} \nabla_{\perp} \varphi\right)-\gamma_{\varphi} \partial_{t} \varphi=\pi N_{F} \frac{\partial \zeta}{\partial A} \partial_{x} A
\end{gathered}
$$

The behavior of different terms in Eq. (17) at small $A(t, x)$ is consistent since at $A \rightarrow 0$ the combination $n-\zeta N_{F}=\Phi N_{F} \rightarrow 0$ by construction and $\gamma_{\varphi} \rightarrow 0$ by definition. Curiously, the Eq. (17) does not show explicitly the commonly assumed longitudinal phase rigidity $\propto \partial_{x}^{2} \varphi$; it is hidden in the first term $\partial_{x}(\pi n-\zeta)$ implicitly, via the relation of $\partial_{x} \varphi+\pi n=0$. This term provides also the driving force for the CDW current which can be written as $-\rho_{c} \partial_{x} \zeta$ instead of the conventionally supposed $\rho_{c} E$ : gradient of the normal carriers chemical potential rather than the electric field.

The second form (18) shows explicitly the physical phase rigidity accumulating all effects of normal carriers and Coulomb interactions. In the first term in the LHS the ratio $\rho_{c} / \rho_{n}$ controls vanishing of the phase rigidity at $A \rightarrow 0$ approaching the metallic state and the Coulomb hardening [54, 61] of the charged phase deformations which dramatically increases with freezing out of screening by normal carriers when $\rho_{n} \rightarrow 0$. Remarkably, the form (18) does not show any driving force for $\varphi$, apart from the less important term in the RHS coming from the gradient of the amplitude $A$. The drive will come only from the boundary conditions for the electric potential transferred to the phase via the local relations of $\partial_{x} \varphi$ and $\Phi$ mediated by $n$.

In the $1 \mathrm{D}$ regime we can exclude the phase by differentiating the Eq. (17) over $x$ to arrive at the closed eq. for the pair of only $n$ and $A$ variables defining the carriers' free energy $F$ :

$$
\begin{aligned}
\gamma \partial_{t} n & =\partial_{x}\left(A^{-2} \partial_{x}\left(N_{F} \partial F / \partial n-n\right)\right) \\
\gamma_{A} \partial_{t} A & =\partial_{x}^{2} A-\partial F / \partial A, F=F(n, A)
\end{aligned}
$$

Imposing only the limit of the electroneutrality while keeping $\sigma_{n}$ to be finite, the driving field for the normal current becomes $-\nabla \mu_{n} \rightarrow-\nabla(\Phi+\zeta-$ 
$\left.n / N_{F}\right)=-\nabla \Phi-\left(\rho_{c} / \rho_{n}\right) \nabla n$, i.e. the effective diffusion coefficient is enhanced as $\rho_{c} / \rho_{n}$ which result could hardly be expected intuitively. In the $1 \mathrm{D}$ regime the diffusion equation and the equation for the phase can be combined and integrated once to yield the equation for the phase alone

$$
\frac{1}{\pi}\left(\frac{1}{\sigma_{C D W}}+\frac{1}{\sigma_{n}}\right) \partial_{t} \varphi+\partial_{x}\left(\frac{1}{\pi} \partial_{x} \varphi+\zeta\right)=\frac{-1}{\sigma_{n}} J(t)
$$

with $\zeta=\zeta(n, A)$ at $n=-\partial_{x} \varphi / \pi$. The RHS of Eq. (19) gives the driving force as the total current $J(t)=j_{c}+j_{n}$ while multiplied by the normal resistance alone. The collective and the normal resistivities combine additively to the effective friction coefficient - the resistivity - of the moving CDW.

\subsubsection{Numerical modeling.}

There are some technical challenges in numerical implementations of the quoted above equations which one commonly does not meet working within conventional GL approaches, see e.g. [42, 62]. The first is the control of compensations at $A \rightarrow 0$ in expressions for total charges, currents, and the condensate energy bringing to action the hidden function of the condensate density $\rho_{c}$. The second is the entanglement in dependencies of thermodynamic functions and their derivatives on $A$ and on $n$ or $\zeta$ : approaching of $n$ or $\zeta$ to critical values should eliminate the energy minimum over $A$ at $A \neq 0$ opening the metallic state, e.g. in the vortex core.

The best, and may be the only analytically transparent, advancing is possible with the simplest Landau type expression for $F$ :

$$
F(n, A)=n^{2} /\left(2 N_{F}\right)+\left(-\tau+\left(n / n_{c r}\right)^{2}\right)\left(A \Delta_{0}\right)^{2} N_{F} / 2+b A^{4} \Delta_{0}^{2} N_{F} / 4
$$

where $a, b \sim 1$. In the Eq. (20) the first term is the contribution of the normal metal, while two other terms give the Landau type expansion in the order parameter with the proximity $\tau \propto\left(1-T / T_{c}\right)$ to the transition temperature being shifted by presence of the normal carriers which critical concentration is $n_{c r}$.

We have performed a numerical modeling employing the following combination of equations: Eq.(13) for $A$ with $F$ given by Eq. (20), Eq.(14) for $\varphi$ and the dissipative equation for $\vec{m}$ generated by the functional (1). The vortices can be spontaneously generated only if equations are written in invariarian variables, rather than in the economical form for the non-unique phase $\varphi$ and angle $\theta$. The order parameter $\vec{\eta}_{s d w}=A \vec{m} \exp (i \varphi)$ is written as $\left.\vec{\eta}_{s d w}=(u+i v)\{p, q\}\right\}$. For the CDW or for frozen alligned spins in 
SDW the invariant variables are just $u, v$. For a SDW with a spin vorticity the allowed varibles were taken as a set of four bilinear combinations $\{\alpha, \beta, \gamma, \delta\}=\{u p, u q, v p, v q\}\}$ imposing the apparent constaint $\alpha \delta=\beta \gamma$. Examples of resulting calculations are shown in Figs.1,2,4a,b.

\section{Conclusions}

A necessity of semi-vortices in conventional antiferromagnets in presence of frozen-in host lattice dislocations was understood already by IED in [13]. In the SDW the semi-vortices become the objects of the lowest energy created in the course of phase slip process; the normal dislocation must split into two objects of the combined topology with the repulsion between them. The combined topological objects where the spin rotations are coupled to DW displacements are stabilized by lowering the Coulomb energy of dislocations. This combination effectively reduces the SDW period allowing e.g. for the twice increase in the NBN frequency, which is an important disputable question.

In presence of spin anisotropy the free rotation of spins is prohibited at large distances from vortices, then the two objects are connected by a string which is the Neel domain wall. Because of the high Coulomb energy of the phase component, the regime of the confinement due to the domain wall is pushed away beyond the distance of the screening length provided by the normal carriers which can be arbitrarily large at low $T$. This allow the combined semi-vortices to be unbound in realistically thin samples.

Exploiting the chiral transformation and understanding the role of the chiral anomaly allows to formulate a phenomenological theory in terms of equations for the DW complex order parameter, the electric potential and the concentration of normal carriers. This approach resolves the problem of violation of the conservation law for condensed carriers rising dangerously for nonstationary inhomogeneous regimes; that allows to model consistently such strongly nonlinear effects as phase slips and nucleation and propagation of phase vortices. Conceptually, the unconventional view is that the collective density and the current always correspond to the nominal number of condensed electrons, as if the temperature is zero and there are no excitations above the gap. The actually present normal carriers are dragged by the phase deformations in such a way that their reaction erases the collective quantities. 


\section{Appendices}

\subsection{Appendix A. Anomalous energy and shape of the charged dislocation in a DW.}

In the Fourier representation, the energy of phase deformations looks like

$$
W\{\varphi\}=\frac{\hbar v_{F}}{4 \pi} \sum\left|\varphi_{k}\right|^{2}\left[C_{\|}^{0} k_{\|}^{2}+C_{\perp} k_{\perp}^{2}+\frac{r_{0}^{-2} k_{\|}^{2}}{\left(k_{\|}^{2}+k_{\perp}^{2}+r_{s c r}^{-2}\right)}\right]
$$

where $r_{0}=\sqrt{\hbar v_{F} s \epsilon /\left(8 e^{2}\right)}$ is the Thomas-Fermi screening length in the parent metal which is the atomic-scale length, and $r_{s c r}=r_{0} / \sqrt{\rho_{n}}$ is the actual screening length by remnant charge carriers in the DW state. Here the normalized normal density $\rho_{n} \rightarrow 1$ at $T \rightarrow T_{c}^{0}$ and $\rho_{n} \sim \exp (-\Delta / T)$ at low $T$ being activated across the DW gap $2 \Delta . \rho_{c}=1-\rho_{n}=C_{\|}^{0}$ is the condensate density. Because of the very large Coulomb energy, the longitudinal gradients are strongly suppressed and we can neglect in (21) all appearances of $k_{\|}$ except for the combination $r_{0}^{-2} k_{\|}^{2}$. At large transverse distances beyond the screening length $r_{\perp} \gg r_{s c r}$ we can neglect also $k_{\perp}^{2}$ in the denominator in 21); then the effective elastic theory is restored, while in stretched coordinates $\left(x \sqrt{C_{\perp} \rho_{n} / \rho_{c}}, r_{\perp}\right)$ :

$$
W\{\varphi\} \approx \frac{\hbar v_{F}}{4 \pi} \sum\left|\varphi_{k}\right|^{2}\left[C_{\|} k_{\|}^{2}+C_{\perp} k_{\perp}^{2}\right], C_{\|}=\rho_{c} / \rho_{n}, k_{\perp} \ll r_{s c r}
$$

Within the screening distance $r<r_{s c r}$, the expression (21) describes a nonanalytic elastic theory, already known for uniaxial ferroelectrics, with the energy dependent on ratio of gradients rather on their values. In this nonscreened regime the energy dependence on the transverse length $L_{\perp}=$ $k_{\perp}^{-1}$ at any given longitudinal scale $L_{\|}=k_{\|}^{-1}$ is not monotonous, showing a minimum at $L_{\perp}^{2} \sim L_{\|} r_{0} C_{\perp}^{1 / 2}$. Multiplying the minimal energy density $\sim C_{\perp} L_{\perp}^{-2}$ by the characteristic volume $L_{\|} L_{\perp}^{2} / a_{\perp}^{2}$ we estimate the energy as a function of $R=L_{\perp}$ as

$$
C_{\perp}^{1 / 2} R^{2} \hbar v_{F} / r_{0}=w_{C} N \text { with } w_{C} \sim T_{c} a_{\perp} / r_{0} \text { at } N=\pi R^{2} / a_{\perp}^{2}
$$

Consider a D-loop of a radius $R$ in the $(y, z)$ plane embracing a number $N=\pi R^{2} / a_{\perp}^{2}$ chains or a D-line stretched in $z$ direction at a distance $Y=a_{\perp} N$ from its counterpart or from the surface. For the DL energy $W_{D}(N)$ the above estimations, with precise values found in [34], can be summarized as

1. $W_{D}(N) \sim T_{c} \sqrt{N} \ln (N)$, valid within length $r_{0}$ - actually only at $N \sim 1$ 
i.e. for a elementary loop embracing only one chain, known as $2 \pi$ soliton. A similar expression is valid at all distances for the spin vortex.

2. $W_{D}(N) \sim N w_{C}, w_{C} \sim T_{c} a_{\perp} / r_{0}$, valid at $r_{0}<R<r_{s c r}$.

3. $W_{D}(N) \sim T_{c}\left(r_{s c r} / r_{0}\right) \sqrt{N} \ln \left(N a_{\perp} / r_{s c r}\right)$ at $R>r_{s c r}$.

Within the wide (at low $T$ ) regime 2. the dislocation energy follows the confinement-type area law $\mathrm{W} \sim N$ rather than the usual perimetrical-logarithmic law $\sim \sqrt{N} \ln N$.

\subsection{Appendix B. The microscopic origin of the chiral anomaly.}

Technically (see [58]), the anomaly appears from uncertainty in calculations of fermionic determinants needing to be regularized, which procedure is usually treated in a relativistically invariant way by traditions of the field theory. It is instructive to see the origin of the anomaly in a transparent way related to rules of many-electronic systems, namely to the Tomas-Fermi procedure. Let the electrons in the parent metal occupy a parabolic spectrum $p^{2} / 2 m$ up to a Fermi energy $E_{F}$. In a smooth potential $V(x)$ the electronic wave functions $\Psi_{E}$ and their density distributions for an eigen energy $E$ are legitimately given by the WKB expressions

$\Psi_{E} \propto\left(E-V(x)^{-1 / 4} \exp \left(i \int d x^{\prime}\left(E-V\left(x^{1 / 2}\right), \rho_{E}=|\Psi(x)|^{2}=\left(E-V(x)^{-1 / 2}\right.\right.\right.\right.$

Summing up $\rho_{E}$, the total density can be obtained and expanded in $V / E_{F}$ as $\rho(x)-\langle\rho\rangle \approx-V(x) N_{F}$ which yields the most important characterization of a metal: the linear response of the density to the applied potential. But if the spectrum linearization is done prematurely, then the Schrödinger eq. becomes the first order one, the wave function $\Psi_{E}$ looses the prefactor, $\rho(x)$ becomes just constant, and the reaction to the local potential disappears. The role of the chiral anomaly action $\left(W_{C A}\right.$ in Eq. (10) is just to restore this missing contribution.

\section{References}

[1] S.A. Brazovskii, I.E. Dzyaloshinskii and N.N. Kirova, Sov. Phys. JETP, 54, 1209 (1981).

[2] S.A. Brazovskii and I.E. Dzyaloshinskii, Sov. Phys. JETP, 44, 1233 (1976). 
[3] S.A. Brazovskii, I.E. Dzyaloshinskii and S.P. Obukhov, Sov. Phys. JETP, 45, 814 (1977).

[4] S. Brazovskii and N. Kirova, Sov. Sci. Reviews, A5, 99, ed. by I.M. Khalatnikov, Harwood Ac. Publ., NY, (1984).

[5] S. Brazovskii, Advances in Theoretical Physics: Landau Memorial Conference, AIP Conference Proceedings 1134, 74 (2009).

[6] I. Dzyaloshinskii, Nobel Symposium 24, 143, Plenum, NewYork (1973).

[7] S.A. Brazovskii, I.E. Dzyaloshinskii and I.M. Krichever, Sov. Phys. JETP, 56, 212 (1982).

[8] S.A. Brazovskii, I.E. Dzyaloshinskii and I.M. Krichever, Phys. Lett. A, 91, 40 (1982).

[9] I.E. Dzyaloshinskii and I.M. Krichever, Sov. Phys. JETP, 56, 908 (1982).

[10] I.E. Dzyaloshinskii and I.M. Krichever, Sov. Phys. JETP, 58, 1031 (1983).

[11] N. Kirova and S. Brazovskii, J. de Physique IV, 9, Pr10-119 (1999).

[12] N. Kirova and S. Brazovskii. J. de Physique, IV, 10, Pr3-183 (2000).

[13] I.E. Dzyaloshinskii, JETP Lett., 25, 98 (1977).

[14] S. Brazovskii and N. Kirova, J. de Physique IV, 9, Pr10-139 (1999).

[15] S. Brazovskii and N. Kirova, Annals of Physics, 403, 184 (2019).

[16] S. Brazovskii and N. Kirova, JETP, 129, 659 (2019).

[17] G. E. Volovik and V. P. Mineev, JETP Lett. 24, 561 (1976).

[18] M. C. Cross and W. F. Brinkman, J. Low Temp. Phys. 27, 683 (1977).

[19] I. A. Lukyanchuk and M. E. Zhitomirsky, Supercond. Rev. 1, 207 (1995).

[20] G. Volovik, PNAS, 97, 2431 (2000).

[21] L. Radzihovsky and A. Vishwanath, Phys. Rev. Lett., 103, 010404 (2009).

[22] S. Brazovskii, Physica B, 404, 482 (2009). 
[23] Y. G. Rubo, Phys. Rev. Lett. 99, 106401 (2007).

[24] V.P. Mineev, Low Temperature Physics, 39, 1056 (2013).

[25] S.I.Anisimov and I.E. Dzyaloshinskii, Sov. Phys. JETP 31 , 773 (1970).

[26] I.E. Dzyaloshinskii and G.E. Volovik, Annals of Physics, 125, 67 (1980).

[27] P. Monceau, Advances in Physics, 61, 325 (2012).

[28] G. Grüner, Rev. Mod. Phys., 60, 1129 (1988).

[29] G. Grüner, Rev. Mod. Phys., 66, 1 (1994).

[30] Charge Density Waves in Solids, eds. L. Gor'kov and G. Grüner, Elsevier Sci. Publ., Amsterdam, 1990.

[31] P. Karpov and S. Brazovskii, Phys. Rev. E 99, 022114 (2019).

[32] S. Brazovskii, J. Supercond. Nov. Magn., 20, 489 (2007).

[33] D. Feinberg and J. Friedel, J. de Physique, 49, 485 (1988).

[34] S. Brazovskii and S. Matveenko, Sov. Phys. JETP, 72, 864 (1992).

[35] M. Hayashi and H. Yoshioka, Phys. Rev. Lett., 77, 3403 (1996).

[36] S. Brazovskii in [30], 425 (1990).

[37] N.P. Ong, G. Varma and K. Maki, Phys. Rev. Lett., 52, 663 (1984).

[38] N.P. Ong and K. Maki, Phys. Rev. B, 32, 6582 (1985).

[39] L.P. Gorkov, JETP Lett., 38, 87 (1983).

[40] L.P. Gor'kov in [30], 403 (1990).

[41] L.P. Gor'kov, Sov. Phys. JETP, 59, 1057 (1984).

[42] I. Batistic, A. Bjelis, L. Gor'kov, J. de Physique, 45, 1049 (1984).

[43] A.I. Larkin and S. Brazovskii, Sol. State Commun. 93, 275 (1995).

[44] S. Brazovskii and T. Nattermann, Advances in Physics, 53, 177 (2004).

[45] T.Yi, N. Kirova and S. Brazovskii, Eur. Phys. J. ST, 222, 1035 (2013). 
[46] T. Yi, A. Rojo Bravo, N. Kirova and S. Brazovskii, J. Supercond. Nov. Mag., 28, 1343 (2015).

[47] S.N. Artemenko, A.F. Volkov, and A.N. Kruglov, Sov. Phys. JETP, 64, $906(1986)$

[48] E. Barthel, G. Quirion, P. Wzietek, D. Jérome, J. B. Christensen, M. Jørgensen and K. Bechgaard, J. de Physique. I., 1, 1501 (1991).

[49] W.G. Clark, M.E. Hanson, S.E. Brown, et al, Synth. Met., 86, 1941 (1997).

[50] H. Requardt, F. Ya. Nad, P. Monceau, R. Currat, J. E. Lorenzo, S. Brazovskii, N. Kirova, G. Grubel and Ch. Vettier, Phys. Rev. Lett., 80, 5631 (1998).

[51] S. Brown and A. Zettle in [30], 223 (1990).

[52] B. I. Ivlev, N. B. Kopnin, Adv. Phys. 3347 (1984).

[53] N. Kopnin, Theory of Nonequilibrium Superconductivity Oxford University Press, Oxford, U.K., 2001.

[54] S. Artemenko and A. Volkov in [30], 365 (1990).

[55] . S. Brazovskii, N. Kirova, H. Requardt, F.Ya. Nad, P. Monceau, R. Currat, J. E. Lorenzo, G. Grubel and Ch. Vettier, Phys. Rev. B 61, (2000) 10640-10650

[56] S. Brazovskii, J. de Physique I, 3, 2417 (1993).

[57] S. Brazovskii, J. Physique IV, 3, 267 (1993).

[58] . I.V.Krive, A.S. Rozhavsky, and V.A. Rubakov, JETP Lett., 46, 121 (1987).

[59] K. Sengupta and N. Dupuis Phys. Rev. B 61, 13493 (2000).

[60] V.M. Yakovenko and H.-S. Goan, Phys. Rev. B 58, 10648 (1998).

[61] B. Hennion, J.-P. Pouget, and M. Sato, Phys. Rev. Lett. 68, 2374 (1992).

[62] S.M. Gol'berg, N.B. Kopnin, and M.I. Tribel'skii, Sov. Phys. JETP 67, 812 (1988). 